\title{
Cost-effective Polythiophene Counter Electrodes for Dye Sensitized Solar Cells
}

\author{
Merve Celik Tolu ${ }^{1, a}$, Buket Bezgin Carbas $^{1}$ and Savas Sonmezoglu ${ }^{2}$ \\ ${ }^{1}$ Karamanoglu Mehmetbey University, Faculty of Engineering, Department of Energy Systems, Karaman, Turkey \\ ${ }^{2}$ Karamanoglu Mehmetbey University, Faculty of Engineering, Department of Metallurgical and Materials, Karaman, Turkey
}

\begin{abstract}
Dye sensitized solar cells (DSSCs) are most promising devices among third-generation solar cells because of low cost, easy production, environmental friendliness, and relatively high conversion efficiency. Counter electrode (CE), which is an important component in DSSCs, functions as an electron transfer agent as well as the regenerator of redox couple. Hitherto, various methods and materials were used to prepare different counter electrodes.Among these materials, conducting polymers have been widely investigated and employed in various applications such as sensors, supercapacitors, energy storage devices, DSSCs and others. In this study, Polythiophene (PTh) conducting polymer was successfully synthesized by electrochemical deposition method, and employed as an alternative to expensive platinum (Pt) CE for DSSC. Besides, PTh conducting polymer was electrochemically deposited via cyclic voltammetry method on FTO substrates. The morphology of the PTh film was characterized by SEM and AFM. Finally, the photovoltaic performance of PTh CE based DSSC was compared with PEDOT CE based device. This new concept—along with promising electrocatalytic activity and facile electron transfer-provides a new approach to enhance the photovoltaic performances of Pt-free DSSCs.
\end{abstract}

\section{Introduction}

The energy required for production of pure silicon is too high so new photovoltaic devices were developed alternative to Si-based solar cells [1]. In 1991, Oregan and Gratzel were developed dye sensitized solar cell (DSSC) and gain new perspective to commercial photovoltaic devices [2]. DSSCs have become developing technology continuously due to their low cost, environmentally friendly fabrication processes, and acceptable energy conversion efficiencies [3-6]. Standart DSSC consist of working electrode (WE/photoanode), liquid electrolyte, and counter electrode (CE/photocathode) [3].

In general, each component performs a specific task and the $\mathrm{CE}$ is one of the most important component in DSSC [3]. Because its task is to reduction of the redox species used as a mediator in regenerating the dye molecules that injected electrons [1,6]. Optimization of cell components are important for cost effective energy production. Over the past few decades, researchers have made consistent progress in replacing components of DSSC with appropriate materials for optimization of device [3].

Until now, Pt was commonly used as CE. Although, $\mathrm{Pt}$ provides a high conductivity and a good catalytic activity, because of its rarity and high cost, researches focus on optimization of $\mathrm{CE}$ with alternative materials [1,3-6]. These alternatives are carbon derivatives, transition metal compounds, and conductive polymers [7-9]. Electrochemical synthesis of conducting polyheterocycles (polypyrrole, polythiophene (PTh), poly $(3,4-$-ethylenedioxythiophene) (PEDOT) etc.) have been reported in literature and some of these studies are about possible application of these polymers in electronic devices such as solar cells [10,11]. Saito et al. reported the preparation of PEDOT on FTO by electrochemical deposition and used as CE in DSSC which is exhibited the efficiency of $3.6 \%$ under $100 \mathrm{~mW} \cdot \mathrm{cm}^{-2}$ [12]. Torabi et al. achieved the highest efficiency of $2.76 \%$ by employing 3-methyl-thiophene (P3MT), synthesized with electrochemical deposition method, as $\mathrm{CE}$ in DSSCs[13]. They examined the improvement of photovoltaic performance as a function of some parameters such as morphology of polymer, duration of polymerization and so on.

In this study, PTh and its derivative PEDOT polymers were successfully synthesized via electrochemical deposition method, and applied as the catalyst material for the counter electrode of a DSSC. Also, their morphological and photovoltaic properties were characterized by AFM as well as SEM and I-V measurements, respectively.

\footnotetext{
a Corresponding author: mervecelik@kmu.edu.tr
} 


\section{Experimental Procedure}

\subsection{Equipments}

The electrochemical measurements were conducted by Ivium compactStat device. The morphology of the conducting polymers was determined using Nanomagnetics Instruments atomic force microscopy (AFM) and JEOL 6390-LV scanning electron microscope (SEM) with an accelerating voltage of $20 \mathrm{kV}$ in the secondary electron image mode. The currentvoltage (I-V) curves of DSSCs were recorded using Keithley 4200 SCS characterization system and standard solar irradiation of $300 \mathrm{Wm}^{-2}$ (xenon arc lamp with AM 1.5 filter) as the light source (Solar Light XPS 300 solar spectrum).

\subsection{Preparation of Counter Electrodes}

Electrodeposition method was used for deposition of PTh and PEDOT conducting polymers on fluorine doped tin oxide (FTO) glass. Three electrode system that consists of FTO glass working electrode (WE), $\mathrm{Ag} / \mathrm{AgCl}$ reference electrode (RE), and Pt sheet as CE were used. Thiophene monomer was dissolved in the medium of dichloromethane, tetrabutylammonium hexafluoro phosphate while EDOT monomer was dissolved in the medium of acetonitrile, lithium perchlorate, and ionic liquid. The oxidation potential of these monomers was found as $2.3 \mathrm{~V}$ for thiophene and $1.4 \mathrm{~V}$ for EDOT via cyclic voltammetry $(\mathrm{CV})$ method. By using this data, polymers were deposited on the electrode via chronoamperometry method with $50 \mathrm{mC}_{\mathrm{cm}} \mathrm{cm}^{-2}$ deposition charge capacity. Finally, the samples deposited on FTO were annealed at $60{ }^{\circ} \mathrm{C}$ for 30 minute in the furnace.

\subsection{Preparation of Photoanodes}

The photoanode was prepared according to our previous report [14]. In briefly, the $\mathrm{TiO}_{2}$ paste (approximately $10 \mu \mathrm{m}$ thickness) were made from 21-nm $\mathrm{TiO}_{2}$ nanopowder in ethanol and printed to FTO substrates using the doctor blade technique and annealed at $450{ }^{\circ} \mathrm{C}$ for $1 \mathrm{~h}$ before sensitization. To fabricate the photoanodes, the $\mathrm{TiO}_{2}$ films were immersed in a dye solution $\left(3.0 \times 10^{-4} \mathrm{M}\right.$ mixture of the ruthenizer 535-bisTBA (N719) in methanol) for $18 \mathrm{~h}$.

\subsection{Preparation of Electrolyte}

The iodine/iodide $\left(\mathrm{I}^{-} / \mathrm{I}_{3}^{-}\right)$electrolyte was prepared according to our previous report [15]. To describe the process briefly, $0.01 \mathrm{M}$ iodine, $0.6 \mathrm{M}$ 1-butyl-3methylimidazolium iodide, $0.1 \mathrm{M}$ 4-tert-butylpridine, and $0.1 \mathrm{M}$ lithium iodide hydrate were prepared in 3methoxypropionitrile solvent, separately. Then, the prepared solutions were mixed and subjected to magnetic stirrer for $2 \mathrm{~h}$.

\subsection{Assembly of DSSCs}

We fabricated the device according to the following method: The photoanode was placed face up on a flat surface, and CEs were placed on top of the photoanode. These two opposing glass plates were offset from one another so that the entire photoanode was covered by the CE. The electrolyte solution was placed at the edges of the plates, and the liquid was drawn into the space between the electrodes via capillary action. We used an epoxy adhesive to hold the electrodes together. Thus, we fabricated the PTh and PEDOT CE based DSSCs having an active area of $\sim 1.0 \mathrm{~cm}^{2}$. The schematic illustration of the DSSC is shown in Figure 1.

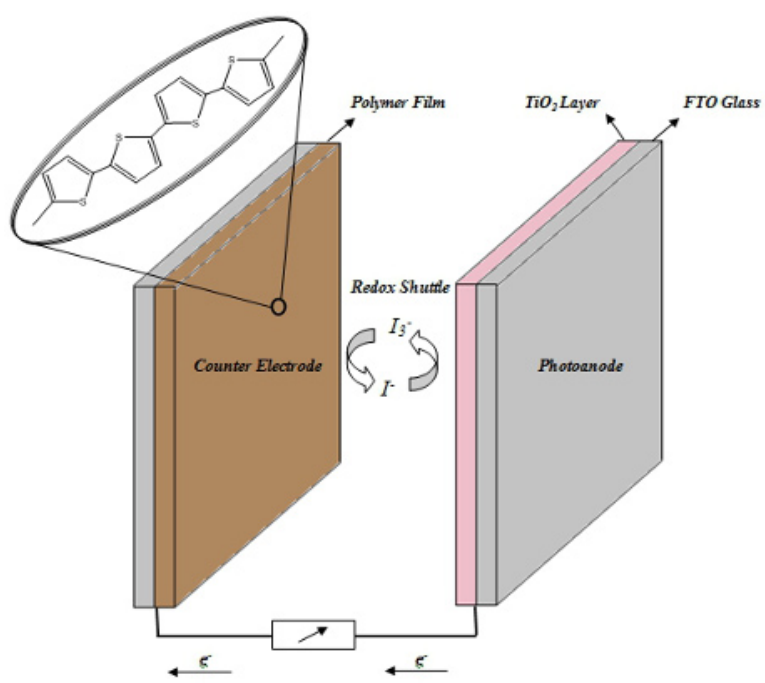

Figure 1. The illustration of DSSC structure with $\mathrm{PTh}-$ and PEDOT-based CEs.

\section{Results and Discussions}

The SEM and AFM images of the PTh and PEDOT polymer deposited films are shown in Figure 2. From the SEM images, it was found that average particle size for PTh and PEDOT conducting polymers is $200 \mathrm{~nm}$ and $400 \mathrm{~nm}$, respectively. From the AFM images, the surface roughness of PTh and PEDOT films was found as $40.37 \mathrm{~nm}$ and $240.80 \mathrm{~nm}$, respectively. Furthermore, all images indicate homogeneous and uniform distributions of grains.

The photovoltaic performances of the DSSCs based on PTh and PEDOT CEs are shown in Figure 3, and the photovoltaic parameters that control the device performance are tabulated in Table 1. The obtained results indicated that conversion efficiency of PTh CE based DSSC $(0.03 \%)$ is lower than PEDOT CE based $\operatorname{DSSC}(4.76 \%)$.

Adhesion of PTh on FTO glass is not good as PEDOT. Therefore, the efficiency of PTh is lower than that of PEDOT. The lower conductivity of PTh and more grain boundaries can be additional reasons of lower efficiency. In future, studies on polymerization medium 
and conditions will be optimized for good adhesion of PTh on FTO.
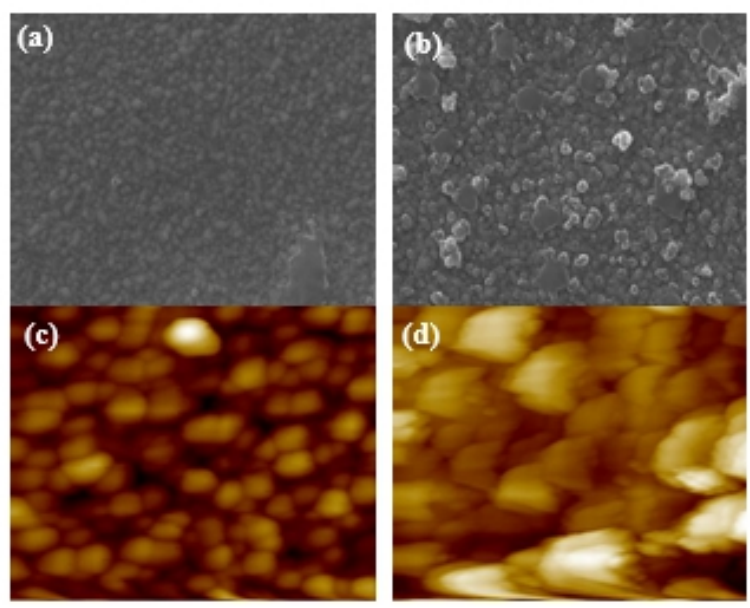

Figure 2. (a,b) SEM images and (c,d) AFM images of PTh and PEDOT films, respectively.
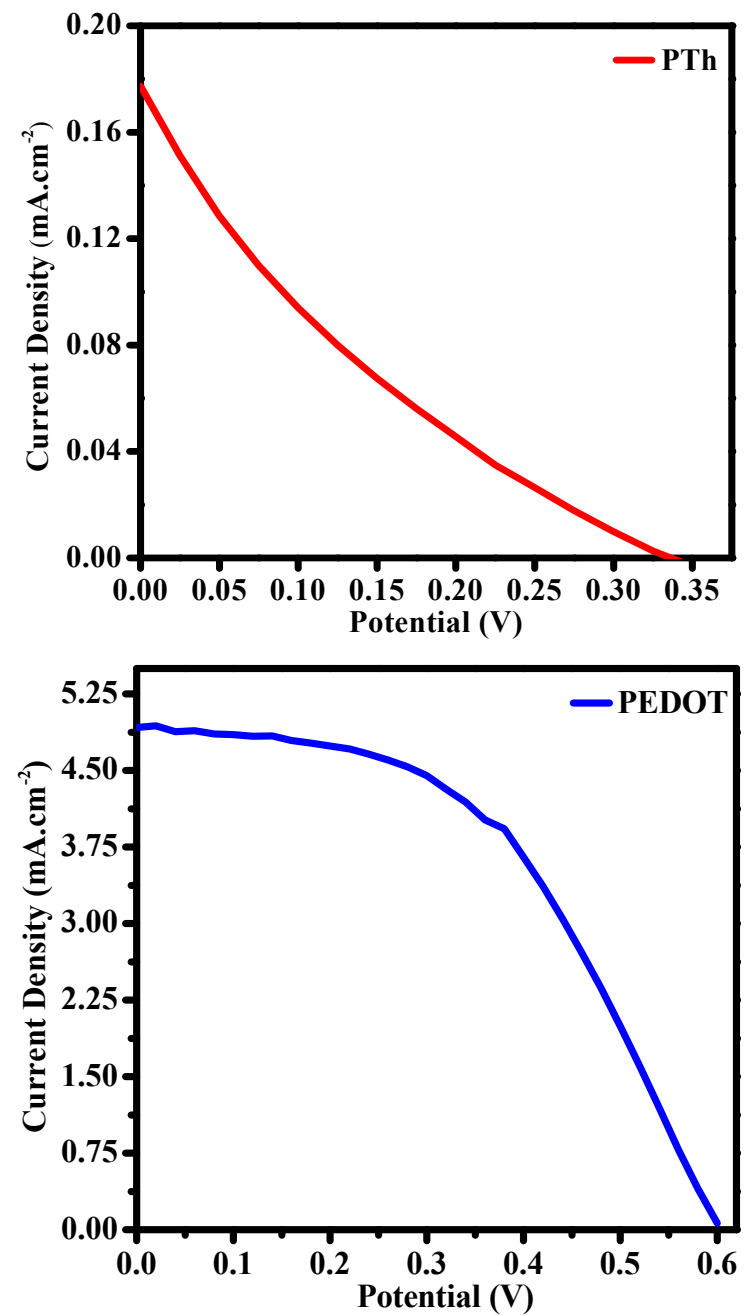

Figure 3. Current density vs. potential plots of PTh and PEDOT CE based DSSCs.
Table 1. The photovoltaic parameters of the conducting polymers CE based DSSCs.

\begin{tabular}{|c|c|c|c|c|}
\hline Devices & $\begin{array}{c}\mathbf{J}_{\text {sc }} \\
(\mathbf{m A . c m}\end{array}$ & $\begin{array}{c}\mathbf{V}_{\text {oc }} \\
(\mathbf{V})\end{array}$ & $\mathbf{F F}$ & $\begin{array}{c}\mathbf{n} \\
(\mathbf{\%})\end{array}$ \\
\hline PTh & 0.18 & 0.33 & 0.17 & 0.03 \\
\hline PEDOT & 4.96 & 0.60 & 0.48 & 4.76 \\
\hline
\end{tabular}

\section{Conclusions}

This study focuses on the fabrication of conducting polymer based CEs in DSSCs. These conducting polymers were synthesized via electrochemical deposition method which is an effective way of polymerization. The polymerized PTh and PEDOT conducting polymers with the same charge density were characterized for their electrochemical and morphological properties. The results suggest that PEDOT based electrode can enhance the catalytic activity between polymer-electrolyte interface of a DSSC. As expected, the power conversion efficiency of DSSC was increased by PEDOT based CE. Efficiency of PTh CE based DSSC is lower than PEDOT CE based device due to the weak adhesion, therefore our studies are still going on to overcome this problem. As a result, DSSC performance can increase by using new polymers creating by substitution of polymer.

\section{References}

1. W. Wei, H. Wang, Y. H. Hu, Int. J. Energy Res. 38, 1099 (2014)

2. B. O'regan, M. Gratzel, Nature, 353, 737 (1991)

3. S. Akın, M. Gülen, S. Sayın, H. Azak, H.B. Yıldız, S. Sönmezoğlu, J. Power Sources, 307, 796 (2016)

4. F. Özel, A. Sarılmaz, B. İstanbullu, A. Aljabour, M. Kuş, S. Sönmezoğlu, Sci. Rep. 6, 29207 (2016)

5. S. Sönmezoğlu, C. Akyürek, S. Akın, J. Phys. D: Appl. Phys. 45, 425101 (2012)

6. S. Sönmezoğlu, V. Eskizeybek, A. Toumiat, A. Avc1, J. Alloys Compd. 586, 593 (2014)

7. Y. Tang, X. Pan, S. Dai, C. Zhang, H. Tian, Key Eng. Mater. 451, 63 (2011)

8. S. Thomas, T.G. Deepak, G.S. Anjusree, T.A Arun, S.V. Nair, A.S. Nair, J. Mater. Chem. 2, 4474 (2009)

9. K. Li, Z. Yu, Y. Luo, D. Li, Q. Meng, J. Mater. Sci. Technol. 23, 577 (2007)

10. S. Sönmezoğlu, R. Taş, S. Akın, M. Can, Appl. Phys. Lett. 101, 253301 (2012)

11. C.T. Li, C.P. Lee, M.S. Fan, P.Y. Chen, R. Vittal, K.C. Ho, Nano Energy, 9, 1 (2014)

12. Y. Saito, W. Kubo, T. Kitamura, Y. Wada, S. Yanagida, J. Photochem. Photobiol. A: Chem. 164, 153 (2004)

13. N. Torabi, A. Behjat, F. Jafari, Thin Solid Films, 573, 112 (2014)

14. R. Taş, M. Gülen, M. Can, S. Sönmezoğlu, Synth. Met. 212, 75 (2015)

15. S. Sönmezoğlu, C. Akyürek, H. Akış, IET Optoelectron. 8, 270 (2014) 